Apidologie, 1974, 5 (3), 271-287.

\title{
SCHALLERZEUGUNG IM DIENST DER VERTEIDIGUNG DES BIENENVOLKES (APIS CERANAi FABR.) *
}

\author{
Une émission sonore au service de la défense de la colonie d'abeilles \\ (Apis cerana Fabr.)
}

\author{
Stefan FUCHS und Nikolaus KOENIGER \\ Zoologisches Institut der TH Darmstadt, Schnittspanstr. 3 \\ Institut für Bienenkunde, Oberursel, Im Rotkopf 5
}

\begin{abstract}
SUMMARY
THE SOUND PRODUCTION, A MEAN OF DEFENCE FOR THE HONEYBEE COLONY (Apis cerana FABR.).

1. Mechanical stimulation of the Apis cerana bee hive releases behaviour which is characterised by a short hissing sound.

2. The sonogramme of the hissing sound shows no harmonic components. The frequencies are mainly between 0,5 and $5 \mathrm{kHz}$.

3. Hissing behaviour can not be released by various chemical and optical stimuli.

4. Airborne sounds (ca. $50 \mathrm{~Hz}$ ) release hissing behaviour most effectivly. With frequencies over $300 \mathrm{~Hz}($ ca. $120 \mathrm{~dB})$ no stimulation is possible.

5. Airborne sounds induce substrate vibrations, which in turn release hissing behaviour.

6. The threshhold for vibration stimuli occurs at a frequency of $100 \mathrm{~Hz}\left(390 \mathrm{~cm} / \mathrm{sec}^{2}\right)$.

7. Hissing behaviour seems to be transmitted from one bee to the other by air movement.

8. The transmission speed is $25 \mathrm{~cm} / \mathrm{sec}$.

9. Aggressivness and the flight activity are significantly reduced after hissing behaviour.

10. Preleminary tests with bears indicate that the hissing sound of the bee colony may be an effective defence against mammals.
\end{abstract}

\section{ZUSAMMENFASSUNG}

1. Durch Erschütterung des Bienenvolkes wird bei Apis cerana ein Verhalten ausgelöst, das durch einen kurzen simultanen Zischlaut gekennzeichnet ist.

* Mit Unterstützung der Deutschen Forschungsgemeinschaft. 
2. Die Analyse des Zischlautes zeigt keine harmonischen Komponenten, der Hauptteil liegt zwischen 0,5 und $6 \mathrm{kHz}$.

3. Das Verhalten läßt sich nicht durch chemische und optische Reize oder sich der Wabe nähernde Insekten (Hornissen, Schmetterlinge) auslösen, wohl aber durch Luftzug.

4. Bei Reizung mit Luftschall waren Frequenzen um $50 \mathrm{~Hz}$ am wirksamsten. Mit Frequenzen über $300 \mathrm{~Hz}$ konnte kein Zischverhalten mehr ausgelöst werden (ca $120 \mathrm{~dB}$ ).

5. Luftschall löst nur mittelbar über Substratschwingung das Verhalten aus.

6. Bei Reizung mit Substratschall liegt die niedrigste Schwelle bei $100 \mathrm{~Hz}\left(390 \mathrm{~cm} / \mathrm{sec}^{2}\right)$.

7. Das Zischverhalten breitet sich von Biene zu Biene über die ganze Wabe aus, die Fortleitungsgeschwindigkeit beträgt $25 \mathrm{~cm} / \mathrm{sec}$.

8. Bei der Übertragung des Verhaltens von Biene zu Biene kann Substratschall ausgeschlossen werden. Auch Körperkontakt ist nicht notwendig. Vielmehr ist die von den Flügeln während des Zischens erzeugte Luftbewegung allein für die Weiterleitung hinreichend.

9. Nach dem Zischverhalten ist die Aggressivität- gemessen an der Anzahl von Angriffsflügen auf eine schwarze Attrappe- reduziert. Auch die Flugtätigkeit ist signifikant kleiner als vor der Zisehreaktion.

10. Vorversuche mit Bären ergaben erste Hinweise auf eine mögliche abschreckende Wirkung des Zischverhaltens auf Säuger.

\section{I. - EINLEITUNG}

Bei den asiatischen Honigbienen, Apis cerana, Apis dorsata und Apis florea, treten verschiedene Verteidigungsverhalten auf, die bei der westlichen Honigbiene (Apis mellifica) nicht vorhanden sind. So wird bei Störung des Apis cerana-Nestes von den Bienen simultan ein kurzer Zischlaut erzeugt. Dieses Verhalten wurde von zahlreichen Autoren beschrieben (BUTLER 1954, Lindauer 1956, Sakagami 1960, Atwal u. Dhaliwal 1969). Deren Beiträge sind jedoch widersprüchlich, Versuche zur biologischen Bedeutung wurden nur selten angestellt (SCHNeider und KLoft 1971).

Wir haben daher erneut den Verhaltensablauf in Bezug auf die Einzelbiene und das Volk, sowie die begleitenden akustischen Erscheinungen untersucht. Zusätzlich wurden Experimente zur Auslösung des Verhaltens, zur Erregungsübertragung von Biene zu Biene und zum Ein fluß des Verhaltens auf mögliche Nesträuber unternommen.

\section{II. - MATERIAL UND METHODEN}

Völker von $A$. cerana werden seit mehreren Jahren im Institut für Bienenkunde in Oberursel gehalten. Die Untersuchungen wurden in den Monaten August-September mit Bienen aus West-Pakistan (North West Frontier Province) durchgeführt. Nach unseren Beobachtungen ergaben sich keine Unterschiede bei Bienen der gleichen Art aus Ceylon, Indien (Poona) und China (Peking). Für die Untersuchungen wurden die Bienen in einem Beobachtungsstock der üblichen Bauart gehalten. Die Kolonie enthielt ca. 12.000 Tiere. Zu den Experimenten wurden die Glasscheiben entweder ganz entfernt oder durch Plastikfolie mit entsprechenden Aussparungen ersetzt. Der Beobachtungsstock war in einem konstant temperierten Raum untergebracht $\left(30^{\circ} \mathrm{C}\right.$ ), der mit einer gelben Glühlampe (Fa. : Radium “ Noninsecta 》) beleuchtet wurde. Über einen Laufkanal von $43 \mathrm{~cm}$ Länge konnten die Bienen in Freie gelangen.

Für Lautstärkebestimmungen wurde ein Brüel u. Kjaer Precision Sound Level Meter verwendet (Typ 2203, Microphone Cartridge 4131/32), dieses konnte an ein Bandgerät ange- 
schlossen werden (UHER-Report 4002, Geschw. $19 \mathrm{~cm} / \mathrm{sec}$ ). Zur Auswertung der Bandaufnahmen diente ein Sonagraph (Kay Sonagraph model 7029 A) und ein Bandpassfilter (KH 3000). Die Aufnahme von Substratschwingungen erfolgte mittels eines geeichten Accelerometers (Bruel u. Kjaer 4332), die Messwerte sind als Beschleunigungsamplituden (SpitzeSpitze) angegeben.

Für Versuche zur Erregbarkeit durch Luftschall wurde die Lautsprecherbox einer Musikanlage (Eigenbau) in $1 \mathrm{~m}$ Entfernung von der Wabe aufgebaut, Sinusschwingungen lieferte ein Tongenerator (HP 200 B). Für Versuche zur Erregbarkeit durch Substratschall benutzten wir einen Schwinger (Derritron VP2), der über einen Verstärker (HP 6832) mit Sinusschwingungen erregt wurde. Der Schwinger war über eine Messingstange durch zwei beidseits der Wabe miteinander verschraubte Blechsterne mit der Wabe verbunden, zur Bestimmung der Schwingungen diente der in $10 \mathrm{~cm}$ Entfernung befestigte Accelerometer.

\section{III. - ERGEBNISSE}

\section{A. - Verhaltensablauf}

Bei mechanischen Erschütterungen des Bienenstockes reagieren die Bienen mit einem kurzen simultanen Zischton (Dauer von 0,5-1 sec, 6 Messungen nach Tonbandaufnahmen, gemessene Dauer 0,$5 ; 0,6 ; 0,5 ; 0,8 ; 1,0$ sec). Die Verhaltensabfolge entspricht der von Sakagami (1960) unter “ shimmering » beschriebenen. Die Bienen führen die Flügel aus der leicht abgespreizten Normalhaltung über dem Körper zusammen, sie stoßen den Körper ruckartig vor, die Bewegung erfolgt ohne Versetzen der Beine. Hierbei entfernt sich das Abdomen von der Wabenoberfläche, wobei es leicht, d.h. um wenige Winkelgrade hin- und herpendeln kann. Dies ist allerdings keine auffällige Komponente des Verhaltens.

Nach dieser Reaktion - bei der auch das Zischen erfolgt — ordnen sich die Tiere mit dem Kopf nach oben an und verharren eine Weile ruhig (ca. 3 sec.), die Flügel werden um ca. $45^{\circ}$ abgespreizt gehalten. Das Volk erscheint während dieser Zeit gegenüber dem aufgelockerten Normalzustand der in mehreren Schichten übereinanderlaufenden Bienen verdichtet und kompakter, die Tiere sind an die Wabe herangezogen. Da das Gesamtverhalten durch das deutliche Geräusch gekennzeichnet ist, soll es im Folgenden als “ Zischverhalten » bezeichnet werden.

Dieses “ Zischen ) wird wohl durch den Flugapparat erzeugt, zumal es im gleichen Augenblick auftritt, in dem die Flügel parallel an den Körper herangezogen werden. Die Lautstärke des abgestrahlten Luftschalls, gemessen in $2 \mathrm{~cm}$ Entfernung von der Wabe, beträgt $65 \mathrm{~dB}$ ( $\mathrm{n}=8$, max. Abweichung \pm $2 \mathrm{~dB}$ ). Das Zischen wurde auf Tonband genommen und im Sonagraphen analysiert (Abb. 1). Das Sonagramm zeigt den geräuschhaften Charakter des Zischens, es lassen sich keine harmonischen Komponenten erkennen. Dieses Geräusch hebt sich erst ab $500 \mathrm{~Hz}$ vom Grundgeräuschpegel ab, der durch umher fliegende Arbeiterinnen erzeugt wird, und reicht dann bis zur oberen Grenze der Tonbandauflösung, wobei der stärkste Anteil zwischen 0,5 und $6 \mathrm{KHz}$ liegt. 

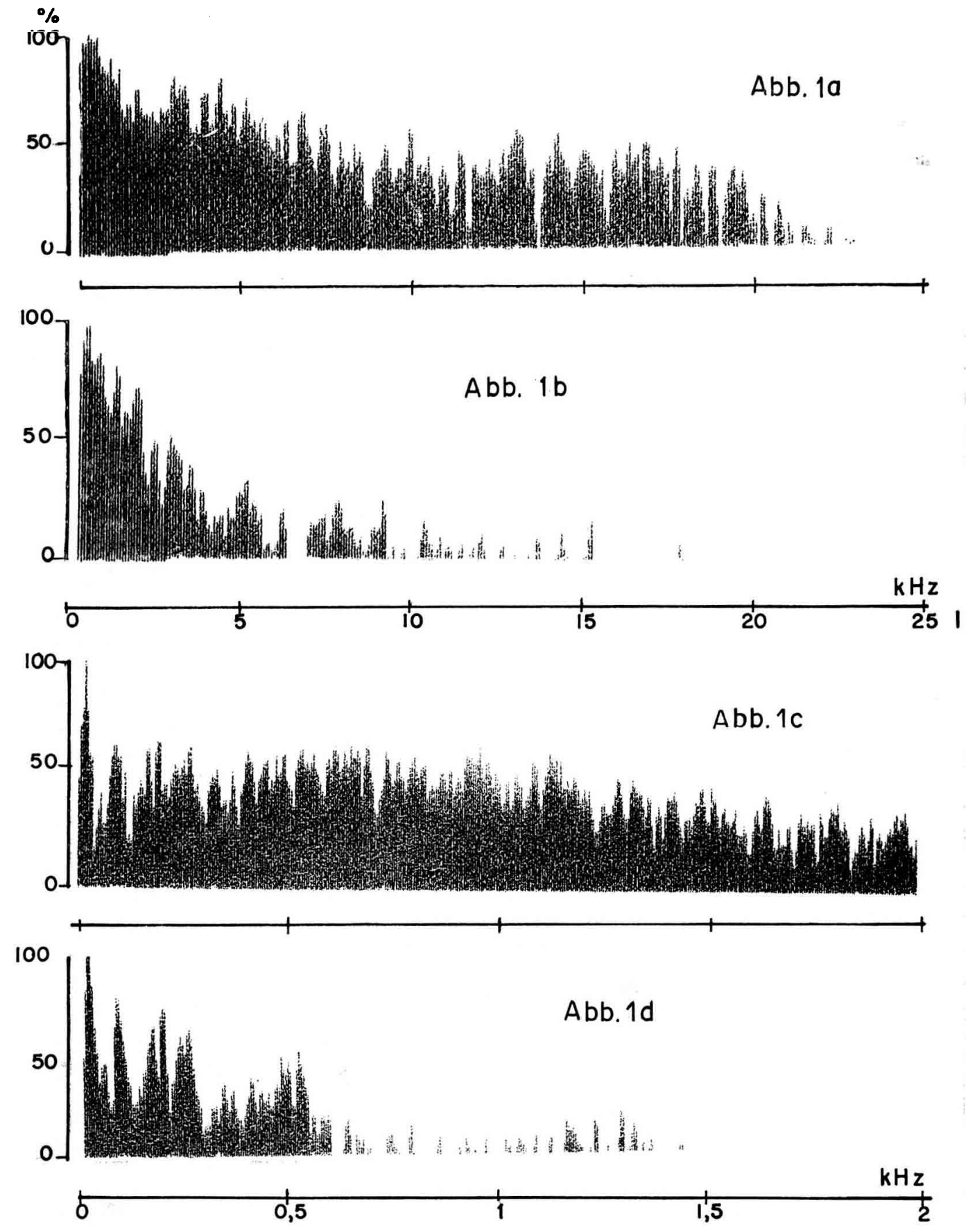

Aвв. 1. - Sonagramm und Bandlini(nspektrum des beim Zischen ven A, cerana entstehenden Luftschalls.

a, b- Bandlinienspektren von $360-36000 \mathrm{H}_{z}$,

a : vom Zischverhalten; $\mathbf{b}$ : vom Grundgeräuschpegel.

c, d - Bandlinienspektren von $20-2000 \mathrm{~Hz}$

c : vom Zischverhalten, d : vom Grundgeräuschpegel.

Auf der Abszisse jeweils die Frequenz, auf der Ordinate die Intensität in \% des Maximums.

FIG. 1. - Sonogramme et spectre sonore du son émis et transmis par l'air dans le sifflement d'Apis cerana.

a et $\mathrm{b}$ - Spectre de 360 à $36000 \mathrm{~Hz}$.

$a$ : dans le comportement du sifflement; $b$ : dans le niveau sonore de base.

c et $\mathrm{d}-$ Spectre de $20-2000 \mathrm{~Hz}$

$c$ : dans le comportement de sifflement; $d$ : dans le niveau sonore de base.

En abscisses la fréquence, en ordonnées l'intensité en \% du maximum 


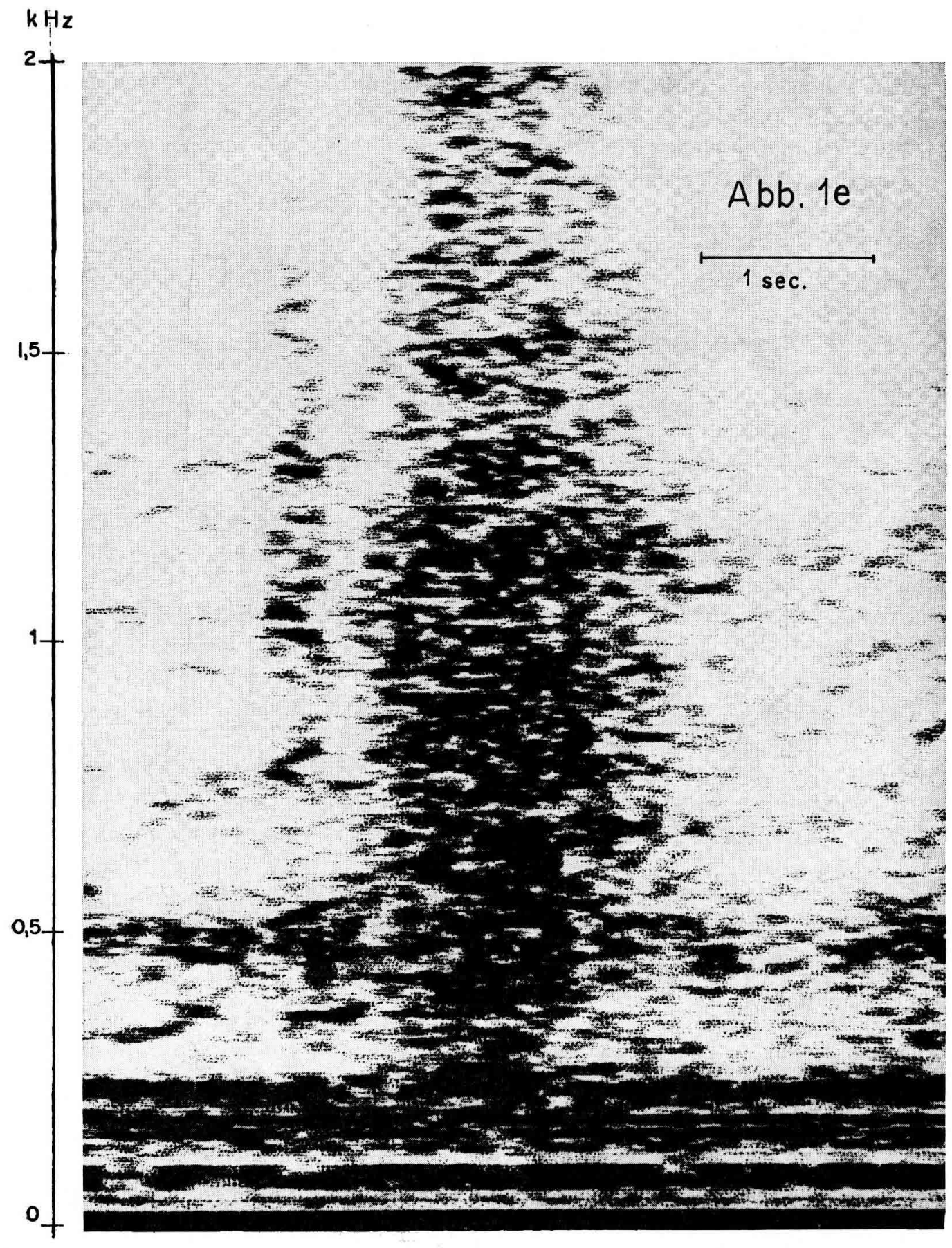

e Sonagramm des Zischverhaltens von $20-2000 \mathrm{~Hz}$, auf der Abszisse der Zeitverlauf, auf der Ordinate die Frequenzen.

e : Sonogramme du comportement de sifflement de 20 à $2000 \mathrm{~Hz}$

En abscisses le temps et en ordonnées les fréquences. 
Während des' Zischtones wird auch die Wabe in Schwingung versetzt. Dieser “ Substratschall 》 wurde mit dem Accelerometer aufgenommen, der in das Wachs eingedrückt und von der Rückseite der Wabe aus verschraubt wurde. Die Intensität wurde am Oszillografen direkt bestimmt, sie betrug im Durchschnitt $80 \mathrm{~cm} / \mathrm{sec}^{2}$ (6 Messungen, max. $110 \mathrm{~cm} / \mathrm{sec}^{2}$, min. $\left.55 \mathrm{~cm} / \mathrm{sec}^{2}\right)$. Zur Ermittlung der Schwingungszusammensetzung wurde ein Bandlinienspektrum aufgestellt (Abb. 2). Es zeigt sich ein scharfes Schwingungsmaximum

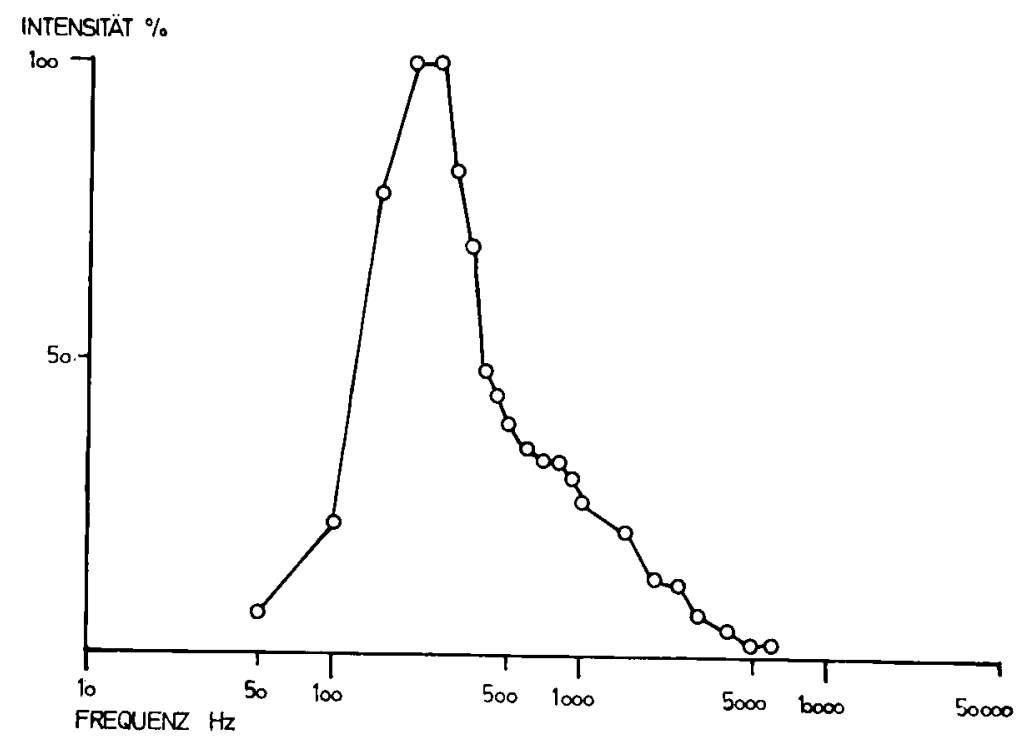

AвB. 2. - Bandlinienspektrum des Substratschalls beim Zischverhalten von A. cerana. Auf der Abszisse die Frequenz, auf der Ordinate die Intensität in \% des Maximums.

FIG. 2. - Spectre sonore du son transmis par le substrat dans le comportement de sifflement d'Apis cerana. En abscisses les fréquences, en ordonnées l'intensité en \% du maximum.

bei $200-250 \mathrm{~Hz}$, dies entspricht einer Resonanzfrequenz der Wabe für erzwungene Schwingungen.

\section{B. - Die Auslösung des Zischverhaltens}

Das Zischverhalten läßt sich leicht durch mechanische Erschütterungen des Nestes auslösen. Angaben über die Abstände des Auftretens bei dauernder Reizung finden sich bei Sakagami (1960) und bei Atwal und Dhaliwal (1969). Sie betragen übereinstimmend bei beiden Autoren 2 bis 8 sec. Es wurden bis zu 14 Reaktionen hintereinander ausgelöst. Die Auslösung durch Luftschall 
ist möglich : Aus $10 \mathrm{~cm}$ Entfernung rückgespielte Tonbandaufnahmen des Zischens (89 dB Lautstärke) lösen das Zischverhalten aus.

Zur Ermittlung der wirksamen Frequenzen wurde mit einer Lautsprecherbox mit reinen Sinustönen jeweils für die Dauer von 1 sec gereizt. Um Klicktransienten beim Einschalten zu vermeiden, wurde der Lautstärkeregler mit der Hand rasch aufgedreht und zurückgenommen, dies entspricht einer Anstiegszeit von ca. $100 \mathrm{~m} / \mathrm{sec}$.

Da Lautstärkemessungen in geschlossenen Räumen problematisch sind, konnte unter diesen Verhältnissen keine genaue Wirksamkeitskurve aufgenommen werden. Es ergab sich, daß das Zischverhalten nur bei Frequenzen unter $100 \mathrm{~Hz}$ gut auslösbar ist (90-100 dB Lautstärke), die Reaktionsschwelle steigt dann schnell an. Ab $300 \mathrm{~Hz}$ war mit unserer Apparatur auch bei sehr hohen Intensitäten (110-120 bB) keine Reaktion mehr auslösbar, es traten lediglich Stopreaktionen auf, wie sie LitTle (1962) für $A$. mellifica beschreibt.

Der Luftschall bringt allerdings auch das Substrat, die Wabe, zum Mitschwingen. Um trennen zu können, ob der Luftschall oder die Schwingung der Unterlage von den Bienen perzipiert wird, wurde die Wabe durch einen Betonklotz (Maße $10+50 \times 15 \mathrm{~cm})$ ersetzt. Mit dem Accelerometer wurde überprüft, daß dieser vom Luftschall nicht mehr zum Mitschwingen gebracht wurde. Erst bei Frequenzen höher als $500 \mathrm{~Hz}$, die zur Auslösung einer Zischreaktion ohnehin nicht mehr in Betracht kommen, traten geringfügige Vibrationen auf.

Die Königin wurde in einem kleinen Käfig in der Mitte befestigt, worauf sich das Volk um sie herum auf dem Betonklotz flächig ausbreitete. Auch bei sehr hohen Lautstärken (über $120 \mathrm{~dB}$ ) ließ sich in 30 Versuchen keine Zischreaktion auslösen, während dies auf andere Weise, etwa durch Anblasen, leicht gelang. Schall ist demnach kein auslösender Reiz, wohl aber die durch ihn verursachte Substratschwingung.

Luftschall setzt sich aus den Komponenten Schalldruck und Schallschnelle zusammen; Schallschnelle ist Bewegung der Luftmoleküle, Schalldruck deren Verdichtung. Aus unserem Versuch kann nicht ausgeschlossen werden, dass Schallschnelle auslösend wirkt. Vor festen Flächen wie dem Betonklotz treten nur Druckknoten auf, die Schallschnelle kommt nicht zur Auswirkung. Es ist zu erwarten, dass vor der immerhin $0,9 \mathrm{~kg}$ schweren Wabe die auftretende Schallschnelle ebenso stark herabgedämpft ist, so dass diese Komponente nicht weiter betrachtet werden muss.

Um festzustellen, welche Substratschallfrequenzen bevorzugt wirksam sind, wurden mit dem Schwinger analog zu den Versuchen mit Luftschall Sinusschwingungen von 1 sec. Dauer auf die Wabe übertragen. Die Intensität wurde schritttweise gesteigert, bis sich eine Zischreaktion auslösen ließ (Abb. 3). 


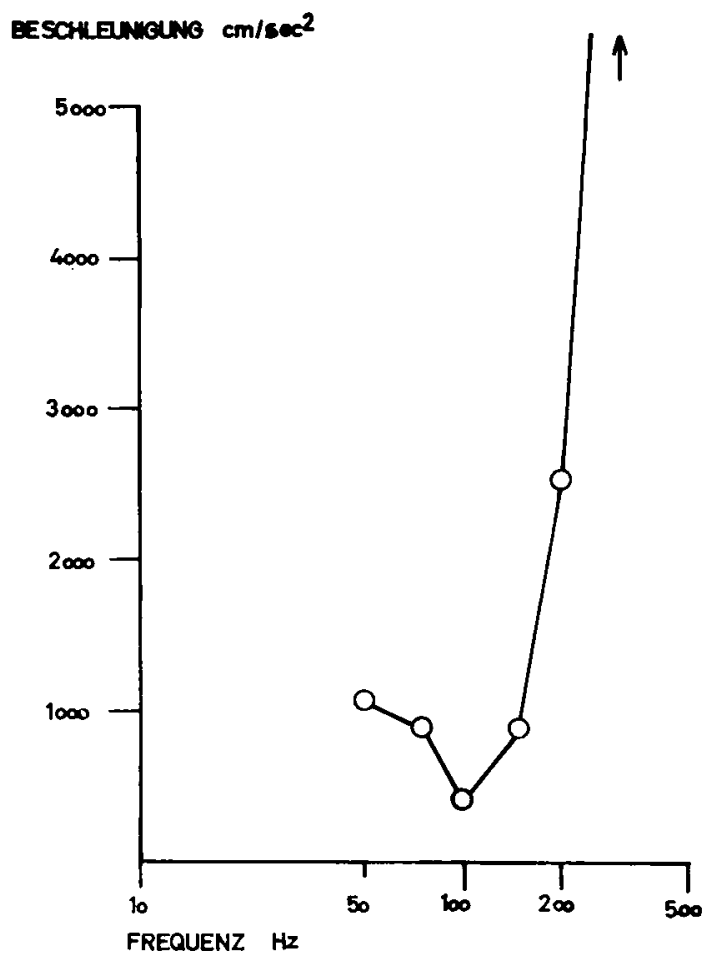

Авв. 3. - Die Auslösbarkeit der Zischreaktion durch Substratschall.

Auf der Abszisse die Frequenz, auf der Ordinate die zur Auslösung einer Reaktion notwendigen Beschleunigungsamplituden (cm/ $\left.\mathrm{sec}^{2}\right)$.

Der Pfeil gibt an, dass bei dieser Intensität keine Reaktion mehr auslösbar war, die Sehwelle also darüber liegt.

Fıg. 3. - Possibilité de déclencher la réaction de sifflement par un son transmis par le substrat. En abscisses la fréquence, en ordonnées l'amplitude d'accélération nécessaire pour déclencher une réaction (en $\mathrm{cm} / \mathrm{sec}^{2}$ ).

La flèche indique qu'avec cette intensité aucune réaction ne pouvait plus être déclenchée, donc que le seuil est au-dessus.

Die niedrigste Reaktionsschwelle lag bei $100 \mathrm{~Hz}\left(390 \mathrm{~cm} / \mathrm{sec}^{2}\right)$, bei $200 \mathrm{~Hz}$ ließ sich die Reaktion nur bei Verwendung sehr hoher Beschleunigungsamplituden, bei $300 \mathrm{~Hz}$ mit dieser Apparatur überhaupt nicht mehr auslösen.

Als besonders gut auslösender Reiz erwies sich Luftbewegung. Schon ein leichtes Anblasen der Bienen wurde mit Zischverhalten beantwortet. Dabei war kalte trockene Luft, die aus einer Flasche gepumpt wurde, etwa ebenso wirksam wie feuchte warme Atemluft.

Ohne Auslösewirksamkeit waren freifliegende Hornissen. Die Tiere flogen 2-10 cm vor der offenen Wabe. Weder durch den Luftzug der Flügel noch durch den optischen Reiz wurde eine Zischreaktion ausgelöst. Auch mellificaBienen, Wachsmotten und andere Schmetterlinge lösten keine Reaktion aus. Dies steht im Widerspruch zu den Beobachtungen von ButLer (1954) und RöPKE (1930), wonach das Verhalten gegen sich nähernde Insekten gerichtet sein soll. 
Chemische Reize, wie Rauch oder Isopentylacetat, dem Alarmpheromon von Apis mellifica, das bei $A$. cerana in gleicher Funktion auftritt (KoEniger, nicht publ.), zeigten keine Auslösewirksamkeit, ebensowenig optische Reize wie bewegte schwarze Scheiben oder Lichtblitze aus einem Elektronenblitzgerät.

\section{C. - Weiterleitung der Zischreaktion}

Die Zischreaktion tritt bei annähernd allen Bienen auf einer Wabe gleichzeitig auf : Dies könnte sich daraus ergeben, dass alle Tiere den auslösenden Reiz gleichzeitig perzipieren. Daneben wird das Verhalten durch einen Kommunikationsmechanismus koordiniert : Bei Reizung durch Anblasen mit einem dünnen Schlauch wird die Zischreaktion zunächst nur bei den unmittelbar betroffenen Bienen ausgelöst, diese erregen dann die benachbarten Tiere, so daß die Reaktion wie eine Welle über die ganze Wabe läuft. Allerdings erfolgt die Weiterleitung nicht in jedem Falle, unter Umständen bleibt die Reaktion auf die direkt betroffenen Tiere beschränkt. Eine Vorzugsrichtung der Erregungsausbreitung vom unteren Teil der Wabe zum oberen, wie sie Sakagami (1960) beschreibt, konnten wir nicht feststellen, die Reaktion breitete sich bei Reizung anderer Teile der Wabe kaum unterschiedlich gut aus.

Die Geschwindigkeit der Erregungsausbreitung bestimmten wir mit zwei Mikrophonen, die in $25 \mathrm{~cm}$ Abstand voneinander dicht vor der Wabe angebracht wurden. Es wurde dann durch gezieltes Anblasen mit einem dünnen Schlauch in der Verlängerung der Verbindungslinie Mikrophon-Mikrophon gereizt. Die Geräusche wurden auf Band genommen und der zeitliche Abstand der Lautstärkemaxima auf einem Oscillografen bestimmt. Aus 15 Messungen ergab sich Abweichungen unter der Genaugkeit des Bestimmungsverfahrens eine Geschwindigkeit von $25 \mathrm{~cm} / \mathrm{sec}$. Berücksichtigt man den minimalen Abstand zwischen zwei Zischreaktionen mit 2 sec. (Sakagami 1960, Atwal u. Dhaliwal 1969), so reicht eine (in sich geschlossene) Laufstrecke von $50 \mathrm{~cm}$ zur Auslösung einer wiederholten Reaktion. Man kann in der Tat beobachten, daß auf diese Weise durch Selbsterregung wiederholte Zischreaktionen zustandekommen.

Zur Untersuchung der Frage, in welcher Weise die Erregung von einer Biene zur anderen weitergegeben wird, wurden drei Hypothesen in Betracht gezogen :

1. durch die Schwingung des Untergrundes,

2. durch direkten Körperkontakt,

3. durch die mit den Flügeln erzeugte Luftbewegung.

\section{zu 1. Substratschwingung}

Die wellenartige Weiterleitung der Zischreaktion durch die Schwingung der Wabe wäre grundsätzlich denkbar, wenn die Abschwächung des Signals 
bei seiner Weiterleitung so gro $\beta$ ist, da $\beta$ immer nur die Tiere in unmittelbarer Umgebung erregt werden könnten. Zur Bestimmung der Abschwächung des Substratschalls wurde eine teilweise mit Honig gefüllte mehrfach bebrütete Wabe mit dem Schwinger angeregt und in verschiedenen Entfernungen vom Ort der Anregung mit einem Plattenspielerkristall abgetastet. Zu jedem Entfernungswert wurden bei Frequenzen des zur Auslösung in Frage kommenden Bereiches $(50,100,200 \mathrm{~Hz})$ je 5 Messungen gemacht und gemittelt (Abb. 4). Bereits in $10 \mathrm{~cm}$ Entfernung ist das Signal um $7 \mathrm{~dB}$ abgeschwächt, es beträgt also etwas weniger als die Hälfte des Ausgangswertes. Die Abschwächung zur Rückseite gegenüber dem Ort der Anregung beträgt 4,5 dB, das Signal hat also etwas mehr als die Hälfte des Ausgangswertes.

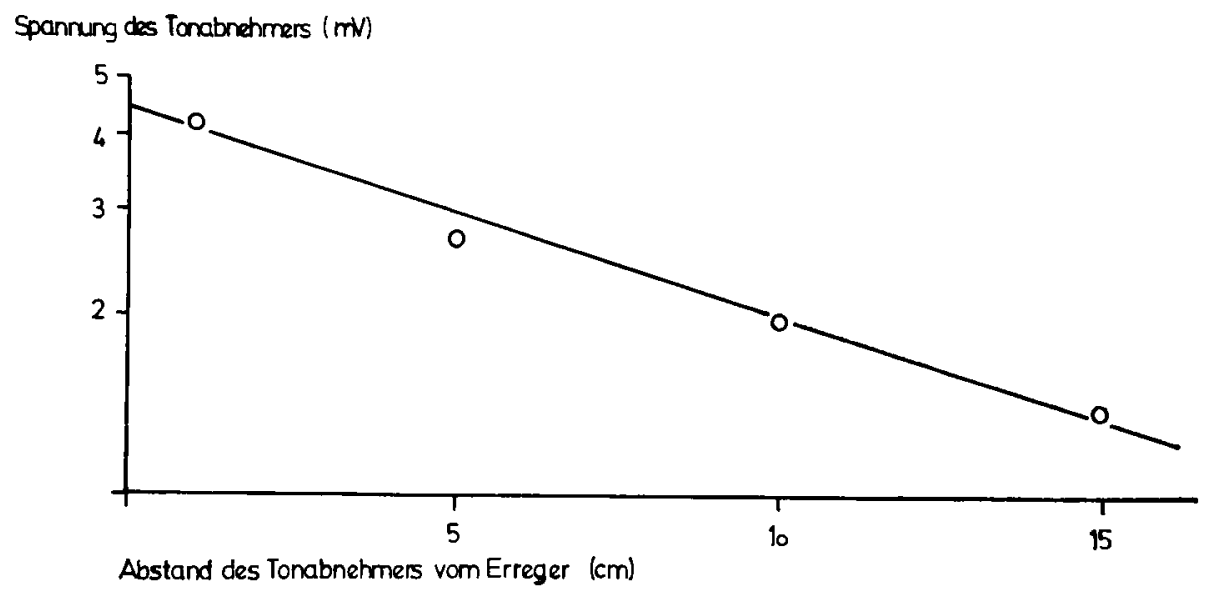

Aвв. 4. - Abschwächung einer honiggefüllten Wabe von A. cerana für Substratschall.

Auf der Abszisse die Entfernung der Messung vom Erregungsort (Tonabnehmer Phillips GP 233), auf der Ordinate die Spannungsabgabe des Tonabnehmers. Die Messpunkte sind aus den Abschwächungen der Frequenzen 50,100 und $200 \mathrm{~Hz}$ gemittelt $(\mathrm{N}=15)$.

FIg. 4. - Affaiblissement d'un rayon d'Apis cerana rempli de miel pour les sons transmis par le substrat. En abscisses distance entre le point d'émission et le point où est faite la mesure (capteur de sons Phillips GP 233), en ordonnées la tension au capteur. Les points de mesure correspondent à des moyennes d'affaiblissement des fréquences 50,100 et $200 \mathrm{~Hz}(\mathrm{~N}=15)$.

Unter diesen Voraussetzungen ist eine wellenartige Ausbreitung der Zischreaktion denkbar. Die elektrophysiologisch bestimmte Empfindlichkeit von A. mellifica für Schwingungen des Untergrundes beträgt nach Autrum und Schneider (1948) $56,1 \mathrm{~cm} / \mathrm{sec}^{2}$ bei $200 \mathrm{~Hz}$, die Beschleunigungsamplituden der Wabe beim Zischverhalten lag zwischen 55 und $110 \mathrm{~cm} / \mathrm{sec}^{2}$. Allerdings lässt sich die Zischreaktion erst durch Beschleunigungsamplituden von minimal $375 \mathrm{~cm} / \mathrm{sec}^{2}$ bei $100 \mathrm{~Hz}$ auslösen, bei Erregung mit rückgespieltem natürlichen Signal lag die Schwelle nicht deutlich tiefer als bei Reizung mit Sinussignalen.

Um zu klären, ob der Substratschall zur Weiterleitung des Verhaltens ausreicht, wurde daher ein weiterer Versuch unternommen : Ein Rahmen aus 
dünnem Holz $(10 \times 20 \mathrm{~cm}$, Höhe $10 \mathrm{~cm})$ auf den auf der Unterseite $1 \mathrm{~cm}$ dicke Schaumgummistreifen geklebt waren, wurde vorsichtig auf die Wabe aufgesetzt und so eine Gruppe von ca. 700 Bienen von den übrigen isoliert. Der Abstand der Bienen innerhalb des Rahmens zu den außerhalb befindlichen betrug $15 \mathrm{~mm}$, die Abschwächung des Substratschalls auf dieser Strecke also höchstens 1/10. Nun wurde durch Anblasen mit einem dünnen Schlauch entweder innerhalb oder außerhalb des Rahmens eine Zischreaktion ausgelöst : Bei 17 Reaktionen außerhalb und 9 Reaktionen innerhalb breitete sich die Reaktion in keinem Fall auf die Tiere jenseits des Rahmens aus. Die Weiterleitung beruht damit nicht auf einer Kommunikation durch Substratschall.

\section{zu 2. Kontakt}

Bei direktem Körperkontakt der Bienen findet die Weiterleitung des Verhaltens statt, die Zischreaktion breitet sich von einer Wabe zur nächsten über eine Distanz von $8 \mathrm{~cm}$ aus, wenn diese durch eine Bienentraube überbrückt wird.

\section{zu 3. Luftzug}

Körperkontakt ist aber keine notwendige Bedingung : In einem Versuch ähnlich dem obigen wurde anstelle eines Rahmens aus Holz ein entsprechender aus Drahtgaze verwendet, um die Unterseite des Rahmens wurde ein dünner mit Phenol getränkter Faden gelegt. Phenol wirkt als Repellent, so daß die Bienen überall einen Abstand von ca. $5 \mathrm{~mm}$ von der Drahtgaze hielten. Bei insgesamt 45 außen durch Anblasen ausgelösten Zischreaktionen griffen 22, also etwa die Hälfte, auf die Bienen innerhalb des Gazerahmens über. Direkter Körperkontakt ist also zur Weiterleitung zwar hinreichend, aber nicht notwendig. Zudem ist nicht ausgeschlossen, daß auch hier Luftzug der verantwortliche Reiz ist. Dies macht die Auslösung nach der dritten Hypothese höchstwahrscheinlich : Die Erregung wird durch die von den Flügeln verursachte Luftbewegung weitergeleitet. Im Gegensatz zu dem Holzrahmen wird diese durch den Drahtgazerahmen nicht behindert. Als Vermittlung wäre hiernach also die Nahfeldschnelle anzusehen. Die im Nahfeld einer Schallquelle auftretende Medienströmung kann erhebliche Schnelleamplituden erreichen, selbst wenn das Schallfernfeld schwach ist (Markl 1973). Die gute Auslösbarkeit des Verhaltens durch Luftzug kommt dieser Deutung entgegen.

\section{D. - Einflu $\beta$ des Zischverhaltens auf die Aggressivität}

Nach Sakagami (1960) und Atwal u. Dhaliwal (1969) soll das Zischverhalten als aggressivitätssteigerndes « Alarm »-Signal wirken. Dies wider- 
spricht unseren Erfahrungen bei der Beobachtung der cerana-Völker. Reagierten die Bienen durch wiederholtes Zischen während der imkerlichen Arbeit, so war die Aggressionsbereitschaft gering. Wurde jedoch ein Volk stärker oder länger gestört, so unterblieb die Zischreaktion und die Bienen wurden immer angriffslustiger. In der dichtgepackten bewegungslosen Stellung, die die Bienen nach dem Zischen einnehmen, erscheint die Aggressivität eher gedämpft, vom Zischverhalten scheint daher ein beruhigender Effekt auszugehen.

Zur Klärung dieser Frage benutzten wir folgende Versuchsanordnung : Parallel zur Wabe pendelte in $25 \mathrm{~cm}$ Abstand eine schwarze Kugel $(\varnothing 10,5 \mathrm{~cm}$ ). Als Maß der Aggressivität wurde die Anzahl der Angriffs flüge innerhalb von 3 min. genommen. Daraufhin lösten wir während 15 sec. Pause die Zischreaktion aus und zählten dann - nach neuerlichem Ingangsetzen des Pendels - die Angriffs flüge während weiterer $3 \mathrm{~min}$. Die Auslösung der Zischreaktion erfolgte durch leichtes Anblasen der Bienen auf der Wabenrückseite, hierdurch wurde vermieden, daß die Aggressivität vom Reiz des Anblasens mitbeein flußt wurde. In insgesamt 18 Versuchen war jedesmal die Anzahl der Angriffsflüge nach dem Zischen vermindert, es griffen vor dem Zischen insgesamt 251 Bienen an, danach nur 107. Um sicherzustellen, daß die Versuchsdauer keine Rolle spielt, wurden 13 Blindversuche ohne Zischreaktion ausgeführt, hier waren es 94 Angriffe in den ersten $3 \mathrm{~min}$., 85 in den folgenden. Der Unterschied zwischen Versuch und Blindversuch ist hochsignifikant $\left(\mathrm{X}^{2}\right.$-Test, $\left.\mathrm{p}<0.01\right)$. Das Zischverhalten übt also einen aggressivitätsdämpfenden Effekt aus.

Des weiteren wurde untersucht, ob das Zischverhalten einen Einfluß auf die Anzahl der das Flugloch verlassenden Bienen hat. In 6 Versuchen verließen während der je 2-minütigen Beobachtungszeiten insgesamt 174. Bienen das Ausflugloch, nach Auslösung des Zischverhaltens im Beobachtungsstock in den folgenden 2 min. nur 39. Auch dieser Unterschied ist hochsignifikant ( $\mathrm{X}^{2}$-Test gegen Gleichverteilung, $\mathbf{p}<0.01$ ).

\section{IV. - DISKUSSION}

Das von uns untersuchte "Zischverhalten » ist wohl das gleiche Verhalten, das Sakagami (1960) und Atwal u. Dhaliwal (1969) unter dem Namen " shimmering » beschreiben. Die Bezeichnung " shimmering " geht auf ButLER (1954) zurück, der es für $A$. cerana beschreibt, doch vermengt er wahrscheinlich verschiedene Verhaltensweisen miteinander, da er von einem starken Schütteln des Abdomens von einer Seite zur anderen berichtet, begleitet von einem eigentümlichen Zischen; dieses Verhalten sei gegen sich annähernde Feinde gerichtet. Ähnlich beschreibt auch RöPKE (1930) ein durch Sammelbienen, Schmetterlinge, Holzbienen und vor allem Hornissen ausgelöstes Verhalten. 
Damit widerspricht auch der Modus der Auslösung unseren Beobachtungen, es scheint daher eher so, daß diese Autoren ein anderes Verhalten meinen, das bei SAKAGAMI (1960) als " abdomen shaking 》 bezeichnet wird und von Kloft und Schneider (1971) als Gruppenverteidigungsverhalten näher untersucht worden ist. Auch dieses Verhalten ist mit einer Lauterzeugung verbunden, einzelne Bienen geben unter Flügelschwirren kurze Tonstöße von 0,3 - 0,7 sec Dauer und 270-298 Hz ab, die in einen langanhaltenden “ Drohton » übergehen könen.

Das von uns untersuchte Verhalten tritt nur im Stock auf der Wabe oder im Schwarm auf, nicht aber bei wenigen versammelten Bienen oder am Nestausgang. Es wird durch Erschütterungen und Luftzug ausgelöst, dagegen nicht durch optische Atrappen, chem. Reize, vorbeifliegende Insekten, wie Hornissen oder durch Lichteinfall. Es breitet sich vom Erregungsort wie eine Welle mit der Geschwindigkeit von $25 \mathrm{~cm} / \mathrm{sec}$ über das ganze Volk aus. Der Luftschall ähnelt einem Zischen. Sein Intensitätsspektrum füllt - bei einem etwas stärkeren Anteil zwischen 0,5 und $6 \mathrm{KHz}$ - in relativ gleichmässiger Verteilung den ganzen Bereich bis zur Grenze der Tonbandwiedergabe bei $20 \mathrm{KHz}$ aus und reicht wohl noch darüber. Diese Frequenzverteilung erinnert an die des « buzz »-Tones, der von Esch und Wilson (1967) für Fliegen beschrieben wurde und der von diesen ebenfalls mit zusammengefalteten Flügeln hervorgebracht werden kann.

Die Wabe als normaler Untergrund schwingt vor allem in einer ihrer Resonanzfrequenzen, in dem von uns untersuchten Fall $200 \mathrm{~Hz}$. Der Reiz, der die Erregung von Biene zu Biene weiterleitet, ist die von den Flügeln erzeugte Luftbewegung, die als Medienströmung im Nahfeld zu betrachten ist (Markl 1973), Substratschall und Schalldruck konnten experimentell ausgeschlossen werden. Unter Körperkontakt ist eine Weiterleitung zwar möglich, doch ist dieser nicht notwendig, wahrscheinlich ist auch hier der Luftzug der verantwortliche Reiz.

Die Aggressivität, gemessen an der Angriffshäufigkeit auf eine schwarze Kugelattrappe, wird durch das Zischverhalten stark gedämpft, die Tiere ordnen sich negativ geotaktisch dichter an die Wabe an. Die Ab flughäufigkeit aus dem Flugloch nimmt deutlich ab. Da sich das Verhalten durch an fliegende Insekten, wie Hornissen, die einen Hauptfeind der Bienen darstellen (TOKUDA 1924), nicht auslösen läßt, gegen die auch andersgeartete Verteidigungsmechanismen bestehen, läßt es sich kaum als Verteidigungsverhalten gegen diese Tiere deuten. Nach den auslösenden Reizen - Erschütterung und Luftzug - könnte es allenfalls gegen Säuger gerichtet sein.

Diese Tiere könnten teils am Honig, teils aber auch an den Nistplätzen (hohle Bäume und Felsspalten) interessiert sein. Auf den Reiz der Erschütterung reagiert das Volk nun mit einem Verhalten, das es optisch unauffälliger 
macht : Die Tiere sitzen ruhig auf der Wabe und die Flugaktivität wird so gut wie eingestellt. Das dabei auftretende Zischen könnte nun als unerwartetes, fremdes Geräusch eine abschreckende Wirkung haben.

Als Honigräuber kommen vor allem Bären in Betracht, von diesen ist zudem bekannt, daß sie sich - geschützt durch ihren dicken Pelz — durch die Angriffe der Arbeiterinnen nicht abwehren lassen. Um einen Hinweis zu erhalten, ob das Zischen einen solchen abschreckenden Effekt haben könnte, stellten wir Vorversuche mit im Herkunftgebiet der Bienen verbreiteten Bären an.

Die Tiere wurden einzeln dressiert, unter einem Holzkasten (40 $\times 22 \times 22 \mathrm{~cm})$ nach einer Honigwabe zu suchen. Dann wurde zusätzlich unter dem Kasten ein Lautsprecher angebracht, über den das cerana-Zischen vom Tonband zurückgespielt werden konnte, sobald der Bär den Kasten berührte. Wegen des hohen Störgeräuschpegels im Zoo erhöhten wir die Lautstärke des rückgespielten Zischens gegenüber dem natürlichen um das etwa Zehnfache. Von den 3 untersuchten Tieren (ein Malaienbär $q$, ein Malaienbär $\hat{\sigma}$ und ein von Menschen aufgezogener, zahmer Kragenbär) reagierte nur das Malaienbär $\delta$ auf das Zischen : Er schreckte zurück und versuchte aus dem Bärenkäfig zu fliehen. Erst nach $15 \mathrm{~min}$. war er bereit, sich dem Kasten wieder zu nähern.

In einem weiteren Versuch spielten wir einem Pandabären das Zischen in seinen Schlafkasten, das Tier floh ins Freigehege auf einen Baum und kam über längere Zeit nicht mehr zurück.

Das Zischen kann damit eine abschreckende Wirkung auf unerfahrene Säuger ausüben. Offen bleibt dabei, ob eine spezifische Wirksamkeit - etwa im Sinne einer Schlangenmimikry — vorliegt. —

Eingegangen im April 1974. Reçu pour publication en avril 1974.

\section{RÉSUMÉ}

\section{Introduction}

Une perturbation d'ordre mécanique de la ruche déclenche chez Apis cerana un comportement qui est caractérisé par un sifflement produit par l'ensemble des abeilles. Le présent travail est une analyse.de ce comportement et des facteurs qui le déclenchent ainsi qu'une expérimentation sur son in fluence sur la fréquence des attaques.

\section{Matêriel et méthode}

Les recherches ont été réalisées en utilisant des colonies d'Apis cerana provenant de l'Ouest du Pakistan (Province de la frontière Nord-Ouest), placées dans des ruchettes d'observation dans un local à température constante $\left(30^{\circ} \mathrm{C}\right)$ et en lumière jaune. Les abeilles pouvaient sortir à l'extérieur grâce à un conduit approprié. 


\section{Résultats}

\section{A. - Comportement.}

Les abeilles réagissent au choc contre la ruche par l'émission simultanée d'un sifflement d'une durée d'environ 0,5 seconde accompagné du comportement suivant : les ailes sont repliées rapidement sur le corps, et l'abdomen est soulevé d'un coup au-dessus du rayon. Puis les abeilles s'alignent toutes avec la tête vers le haut du rayon et tiennent leurs ailes écartées de telle sorte que les axes des deux paires d'ailes forment un angle d'environ $90^{\circ}$. Cette posture est conservée par les abeilles pendant environ 3 secondes avant le retour à l'état « normal » qui est plus relâché.

L'intensité sonore du son transmis par l'air, mesurée à $2 \mathrm{~cm}$ de distance du rayon est de $65 \mathrm{~dB}$.

Le sonogramme (Fig. 1) montre le caractère acoustique du sifflement qui est exempt d'harmoniques. Pendant le sifflement il se produit des vibrations sur le rayon. L'intensité de ces vibrations du substrat a été déterminée directement au moyen d'un accéléromètre branché sur une oscillographe. Elle était d'environ $80 \mathrm{~cm} / \mathrm{sec}^{2}$. Le spectre des vibrations du substrat (Fig. 2) montre un net maximum à $200-250 \mathrm{~Hz}$, ce qui correspond à une fréquence de résonance du rayon.

\section{B. - Déclenchement.}

Son transmis par l'air. Le déclenchement du comportement de sifflement par un son transmis par l'air est possible. Les enregistrements du sifflement sur bande magnétique ré-émis à $10 \mathrm{~cm}$ de distance sous $89 \mathrm{~dB}$, déclenchent le comportement de sifflement. En utilisant comme moyen d'excitation des sons sinusoïdaux de différentes fréquences on a trouvé que seules les fréquences inférieures à $100 \mathrm{~Hz}(90-100 \mathrm{~dB})$ sont efficaces. A partir de $300 \mathrm{~Hz}$, même pour de très fortes intensités il est impossible de déclencher le comportement de sifflement.

D'autres expériences ont été réalisées au moyen d'un dispositif modifié. Des abeilles ont été placées sur un bloc de béton de $50 \times 50 \times 15 \mathrm{~cm}$ et excitées par un son aérien. Même pour de très fortes intensités sonores, les sons transmis par voie aérienne vers le béton ne provoquent aucune réaction alors que, dans les mêmes conditions, on peut provoquer le sifflement très facilement en soufflant sur les abeilles. Par conséquent l'excitation sonore ne déclenche pas le sifflement mais, par contre, la vibration du substrat consécutive à la réception du son le déclenche.

2) Son transmis par le substrat. Au moyen d'un vibreur, des fréquences sinusoïdales ont été transmises au rayon (Fig. 3). Le seuil inférieur de réaction était aux environs de $100 \mathrm{~Hz}$ $\left(390 \mathrm{~cm} / \mathrm{sec}^{2}\right)$. Au-dessus de $300 \mathrm{~Hz}$ il était impossible de déclencher le comportement de sifflement même en utilisant des amplitudes d'accélération très élevées.

3) Mouvement de l'air. Un souffle léger sur les abeilles suffit à provoquer le comportement de sifflement. L'air froid et sec pompé dans un flacon est à peu près aussi efficace que l'air chaud et humide de la respiration.

Différentes excitations chimiques et optiques se sont révélées dépourvues d'effet sur le déclenchement du comportement de sifflement.

\section{C) - Transmission de la réaction de siffement.}

En soufflant sur les abeilles au moyen d'un tube très fin on peut déclencher le comportement de sifflement chez les seules abeilles qui sont directement atteintes. Celles-ci excitent par leur sifflement les abeilles voisines et ainsi la réaction se propage comme une onde sur tout le rayon. Au moyen de 2 microphones on a pu mesurer sa vitesse de propagation qui est de $25 \mathrm{~cm}$ par seconde. 
Pour répondre à la question de savoir comment l'excitation se transmet d'une abeille à l'autre on a réalisé différentes expériences.

1) Un cadre de bois $(10 \times 20 \mathrm{~cm}$, hauteur $10 \mathrm{~cm})$ a été déposé avec précaution à la surface du rayon et, de cette façon, environ 700 abeilles ont été isolées du reste de la colonie. Puis, en soufflant sur les abeilles, soit à l'intérieur soit à l'extérieur du cadre, on a provoqué le comportement de sifflement. Au cours de 26 essais on a constaté qu'en aucun cas la réaction ne se propage de l'autre côté du cadre. On en conclut que la transmission ne repose pas sur une communication au moyen de sons transmis par le substrat.

2) A la place du cadre de bois on a utilisé de la toile métallique en faisant bien attention de respecter la même distance entre les abeilles à l'intérieur et à l'extérieur du cadre que dans l'expérience précédente effectuée avec le cadre de bois. Sur 45 essais on a obtenu 22 réactions positives, c'est-à-dire que l'excitation a été transmise à travers la toile métallique. On peut en conclure que l'excitation est transmise par le mouvement de l'air provoqué par les ailes, ce qui confirme la facilité avec laquelle on peut déclencher le comportement de sifflement en soufflant sur les ailes.

\section{D) - Influence du comportement de siflement sur l'agressivité.}

Pour éclaircir cette question on a utilisé le dispositif suivant : parallèlement au rayon et à une distance de $25 \mathrm{~cm}$ on actionne un pendule constitué par une boule noire à l'extrémité d'un fil. Comme mesure de l'agressivité on utilise le nombre de vols d'attaque en 3 minutes. Ensuite on arrête le pendule pendant 15 secondes et pendant cette pause on déclenche la réaction de sifflement. Les vols d'attaque pendant les 3 minutes qui suivent et après remise en route du pendule sont comptées. Dans les 18 essais effectués le nombre des attaques a diminué à chaque fois après le sifflement. Avant le sifflement 151 abeilles au total ont attaqué alors qu'après le sifflement on n'en a compté que 107. Pour s'assurer que la durée de l'expérience ne joue aucun rôle on a fait 13 essais à blanc c'est-à-dire sans réaction de sifflement. La différence entre essais et essais à blanc est hautement significative $\left(\mathrm{X}^{2}, \mathrm{p} .>0,01\right)$.

\section{Discussion}

Le comportement de sifflement étudié ici a été décrit par Sakagami (1960) et Atwal et Dhaliwal (1969) sous le nom de " shimmering ». Cette dénomination vient de Butler (1954, pages 14-15) : "When a hornet and wax moth or other intruder approaches the nest, the bees shake their bodies violently from side to side in concert, a behaviour pattern which I called the shimmering bahaviour of Apis indica (syn. cerana). "

Il y a donc de très grandes différences aussi bien dans le déroulement du comportement que dans le mode de déclenchement entre " shimmering » et le comportement étudié ici et que nous avons appelé comportement de sifflement (“Zischverhalten »).

Le comportement de sifflement n'intervient que dans la ruche, sur le cadre ou dans l'essaim. Il est déclenché par les chocs et les jets d'air mais pas par les leurres optiques, les excitations chimiques, les insectes volant à proximité ni la lumière. Il se propage à partir du point d'excitation comme une onde à la vitesse de $25 \mathrm{~cm}$ par seconde dans toute la colonie. L'agressivité, mesurée à travers la fréquence des attaques sur une boule noire servant de leurre, est abaissée par le comportement de sifflement. 'Tout cela permet de penser qu'il s'agit d'un moyen de défense contre les Mammifères. Ces animaux peuvent être intéressés soit par le miel soit par la cavité de nidification.

Comme voleurs de miel,' les ours sont à considérer avant tous les autres car, protégés par leur épaisse fourrure ils ne se laissent pas intimider par les attaques des abeilles. A fin d'avoir des indications dans cette direction, nous avons réalisé des expériences préliminaires avec des ours vivant dans les pays d'origine d'A pis cerana. Les animaux ont été dressés individuellement 
à chercher un rayon de miel sous une caisse de bois. Puis, on a introduit en plus sous la caisse, un haut-parleur émettant le son sifflant d'Apis cerana aussitôt que l'ours touche à la caisse. En raison du haut niveau sonore dans le zoo nous avons multiplié par 10 environ l'intensité sonore du sifflement enregistré. Sur trois animaux étudiés, seul réagit un ours de Malaisie. Il eut peur et chercha à s'enfuir de sa cage. Ce n'est qu'après 15 minutes qu'il s'approcha de nouveau de la caisse. Dans une autre expérience nous avons émis le sifflement dans la niche d'un petit panda; l'animal s'enfuit dans l'enclos et ne revint que longtemps après.

Le sifflement peut donc exercer une action d'effroi sur les ours. Il resterait à déterminer si l'on est en présence d'une activité spécifique, par exemple dans le sens d'un mimétisme du serpent.

\section{LITERATURVERZEICHNIS}

Autrum H., und Schneider W. : Vergleichende Untersuchungen über den Erschütterungssinn der Insekten. Z. vergl. Physiol. 31, 77-88 (1948).

Atwal, A.S. und Dhaliwal, G.S. : Some behavioural characteristics of Apis indica F. and A. mellifica L. Indian Bee Journal 31, 1-8 (1969).

Butler, C.G. : The World of the Honeybee. London (1954).

Esch, H. und Wilson, D : The sounds produced by flies and bees Z. vergl. Physiol. 54, 256-267 (1967).

Koeniger, N. und Fuchs, S. : Kommunikative Schallerzeugung von Apis cerana Fabr. im Bienenvolk. Naturwissenschaften 4, 169 (1972).

Lindauer, M. : Über die Verständigung bei indischen Bienen. Z. vergl. Physiol. 38, 521-575 (1956).

LitTLE, H.F. : Reactions of the Honey Bee, Apis mellifera L., to artificial Sounds and Vibrations of Known Frequencies Ann. of the Ent. Soc. of America 55, 82-98 (1962).

MARKL, H. : Leistungen des Vibrationssinnes bei wirbellosen Tieren. Fortschr. der Zool. 21, Heft $2 / 3$ 101-118 (1973).

Roеpкe, W. : Beobachtungen an indischen Bienen. Med. Landbouwhoogescool Wageningen 34, 1-33 (1930).

Schneider, P. und Kloft, W. : Beobachtungen zum Gruppenverteidigungsverhalten der Östlichen Honigbiene Apis cerana Fabr. Z. Tierpsychol. 29, 337-342 (1971).

Sakagami, F. : Preliminary Report on the specific Difference of Behaviour and other ecological Characters between european and japanese Honeybees. Acta Hymenopterologica 1, 71-198 (1960).

ToKuna. Y. : Studies on the honeybee. Tr. sapporo Nat. Hist. Soc. 9, 1 (1924). 